\title{
Erratum to: The effects of a life goal-setting technique in a preventive care program for frail community-dwelling older people: a cluster nonrandomized controlled trial
}

Yoshimi Yuri ${ }^{1,2 *}$, Shinichi Takabatake ${ }^{2}$, Tomoko Nishikawa ${ }^{2}$, Mari Oka ${ }^{3}$ and Taro Fujiwara ${ }^{4}$

\section{Erratum}

After the publication of this work [1] an error was discovered in the calculation of the sample size. This error does not substantially alter the results or conclusions. The following paragraph in the section 'Sample size and Power' has been modified as follows:

\section{Original text.}

Based on a previous report [29], we expected to demonstrate a difference of at least $7 \%$ in mean change in score of the frailty checklist between the intervention and control groups (equivalent to an effect size of 0.43). Based on a power of $80 \%$ and an alpha of 0.05 , we decided on a minimum sample size of $n=32$ per group (64 in total). Based on expected drop-out rate of $50 \%$ in the control group and $60 \%$ in the intervention group, the required sample size was $n=64$ for the control group and $n=80$ for the intervention group ( $n=144$ in total).

\section{Revised text.}

Based on a previous report [29], we expected to demonstrate a difference of at least $20 \%$ in mean change in score of the frailty checklist between the intervention and control groups (equivalent to an effect size of 0.41). Based on a power of $80 \%$ and an alpha of 0.05 , we decided on a minimum sample size of $n=47$ per group (94 in total). Based on an expected drop-out rate of $30 \%$ in the control group and $40 \%$ in the intervention group, the required sample size was $n=67$ for the control group and $n=78$ for the intervention group ( $n=145$ in total).

It was also discovered after publication that there was a numerical error in the 3rd row, 4th column of table 5. The entry " $21.94 \pm 4384$ " should be " $21.94 \pm 4.84$ ".

\section{Author details}

${ }^{1}$ Department of Rehabilitation Sciences, Faculty of Allied Health Sciences, Kansai University of Welfare Sciences, Osaka, Japan. ${ }^{2}$ Graduate School of Comprehensive Rehabilitation, Osaka Prefecture University, Osaka, Japan. ${ }^{3}$ Elderly Care Office, Izumi, Osaka, Japan. ${ }^{4}$ Izumi Rehabilitation Home-Visit Nursing Care Station, Osaka, Japan.

Received: 21 April 2017 Accepted: 21 April 2017

Published online: 08 May 2017

Reference

1. Yuri Y, Takabatake S, Nishikawa T, Oka M, Fujiwara T. The effects of a life goal-setting technique in a preventive care program for frail communitydwelling older people: a cluster nonrandomized controlled trial. BMC Geriatr. 2016:16:101.

* Correspondence: yuri@tamateyama.ac.jp

${ }^{1}$ Department of Rehabilitation Sciences, Faculty of Allied Health Sciences,

Kansai University of Welfare Sciences, Osaka, Japan

${ }^{2}$ Graduate School of Comprehensive Rehabilitation, Osaka Prefecture

University, Osaka, Japan 